POS $\quad$ PROCEEDINGS

\title{
New results on spin and 3D structure measurements
}

\author{
Haiyan Gao*t \\ Department of Physics, Duke University, Durham, North Carolina 27708, USA \\ Duke Kunshan University, Kunshan, Jiangsu 215316, China \\ E-mail: gao@phy.duke.edu \\ Tianbo Liu \\ Department of Physics, Duke University, Durham, North Carolina 27708, USA \\ Thomas Jefferson National Accelerator Facility, Newport News, VA 23606, USA \\ E-mail: liutb@jlab.org
}

\section{Zhiwen Zhao}

Department of Physics, Duke University, Durham, North Carolina 27708, USA

E-mail: zhiwen.zhao@phy.duke.edu

\begin{abstract}
The nucleon spin structure has puzzled the physics community for about 30 years since the original discovery made by the EMC collaboration in late 1980s that the quark spin only contributes a small fraction to the nucleon spin. With major efforts from both theoretical and experimental sides, the quark spin contribution is relatively well known and the gluon spin contribution is becoming known in recent years. To fully understand the nucleon spin structure, the study has gone beyond one dimensional to three dimensional (3D) to probe the confined motion and access the orbital angular momentum of the partons inside the nucleon. In this paper, we briefly review the experimental status and the progress during the last year on the study of nucleon spin and 3D structures.
\end{abstract}

XXVI International Workshop on Deep-Inelastic Scattering and Related Subjects (DIS2018)

16-20 April 2018

Kobe, Japan

\footnotetext{
* Speaker.

${ }^{\dagger}$ This work is supported in part by the U.S. Department of Energy, Office of Science, Office of Nuclear Physics under contract Nos. DE-FG02-03ER41231 and DE-AC05-06OR23177.
} 


\section{Introduction}

Quantum chromodynamics (QCD) is nowadays viewed as the fundamental theory of strong interactions. The asymptotic freedom that the strong coupling constant is small at high energy scales is one of the main properties of QCD. It ensures the application of the perturbation theory and has been precisely tested in experiments. On the other hand, QCD at low energy scales, e.g. the hadron scale, remains unsolved due to its nonperturbative nature. It is listed as one of the top 10 challenges for physics, and is also important for discovering new physics beyond the standard model.

The nucleon structure is one of the most active areas in QCD. In late 1980s, the EMC collaboration firstly measured the quark spin contribution in a polarized nucleon and found that it only carried a small fraction $[1,2]$. This result severely contradicts with the naive quark model in which the nucleon spin is all carried by the quark spin, and it was referred to as the "proton spin crisis". It is now known that the quark spin, $\frac{1}{2} \Delta \Sigma$, contributes about $1 / 3$ of the nucleon spin, and the remaining nucleon spin is attributed to the gluon spin, $\Delta G$, and orbital angular momenta, $L_{q}$ (quarks) and $L_{g}$ (gluons), as

$$
S_{N}=\frac{1}{2}=\frac{1}{2} \Delta \Sigma+\Delta G+L_{q}+L_{g},
$$

although the decomposition is theoretically nontrivial and one may have different operator definitions of the orbital angular momenta [3, 4].

In last 30 years, impressive experimental progress has been made in QCD spin physics, including inclusive spin-dependent deep inelastic scattering (DIS) experiments at CERN (EMC, SMC, and COMPASS), SLAC (E80, E142, E143, E154, and E155), DESY (HERMES), and JLab (Hall A, B, and C), semi-inclusive DIS experiments at CERN (SMC and COMPASS), DESY (HERMES), and JLab (Hall A, B, and C), polarized $p p$ collisions at BNL (PHENIX and STAR) and FNAL (POL. DY), and polarized $e^{+} e^{-}$collisions at KEK (Belle). In this paper, we briefly review the status and the progress in the last year on nucleon spin and 3D structures.

\section{Progress in 1D spin structures}

Among the four terms in the nucleon spin decomposition, the quark spin is the most extensively studied. A global QCD analysis of the inclusive DIS, semi-inclusive DIS (SIDIS), and the single inclusive $e^{+} e^{-}$data to simultaneously fit polarized parton distribution functions (PDFs) and charged pion and kaon fragmentation functions (FFs) is performed by the JAM Collaboration using the iterative Monte Carlo approach [5], shown in Figure 1. At the scale $Q=1 \mathrm{GeV}$, the extracted total quark spin is $\Delta \Sigma=0.36(9)$, the triplet axial charge is $a_{3}=\Delta u_{+}-\Delta d_{+}=1.24(4)$, and the octet axial charge is $a_{8}=\Delta u_{+}+\Delta d_{+}-2 \Delta s_{+}=0.46(21)$. Without imposing the $\mathrm{SU}(3)$ symmetry in the analysis, a small value of the strange quark polarization is extracted with relatively large uncertainty $\Delta s_{+}=-0.03(10)$.

In DIS, the gluon spin-dependent PDF can be studied via the scaling violation of the spin structure function $g_{1}\left(x, Q^{2}\right)$ provided wide $Q^{2}$ span of the data, but only loose constraints are obtained. A measurement of asymmetries directly sensitive to the gluon polarized PDF is one of primary motivations of the RHIC spin program, and the longitudinal double spin asymmetries of inclusive 

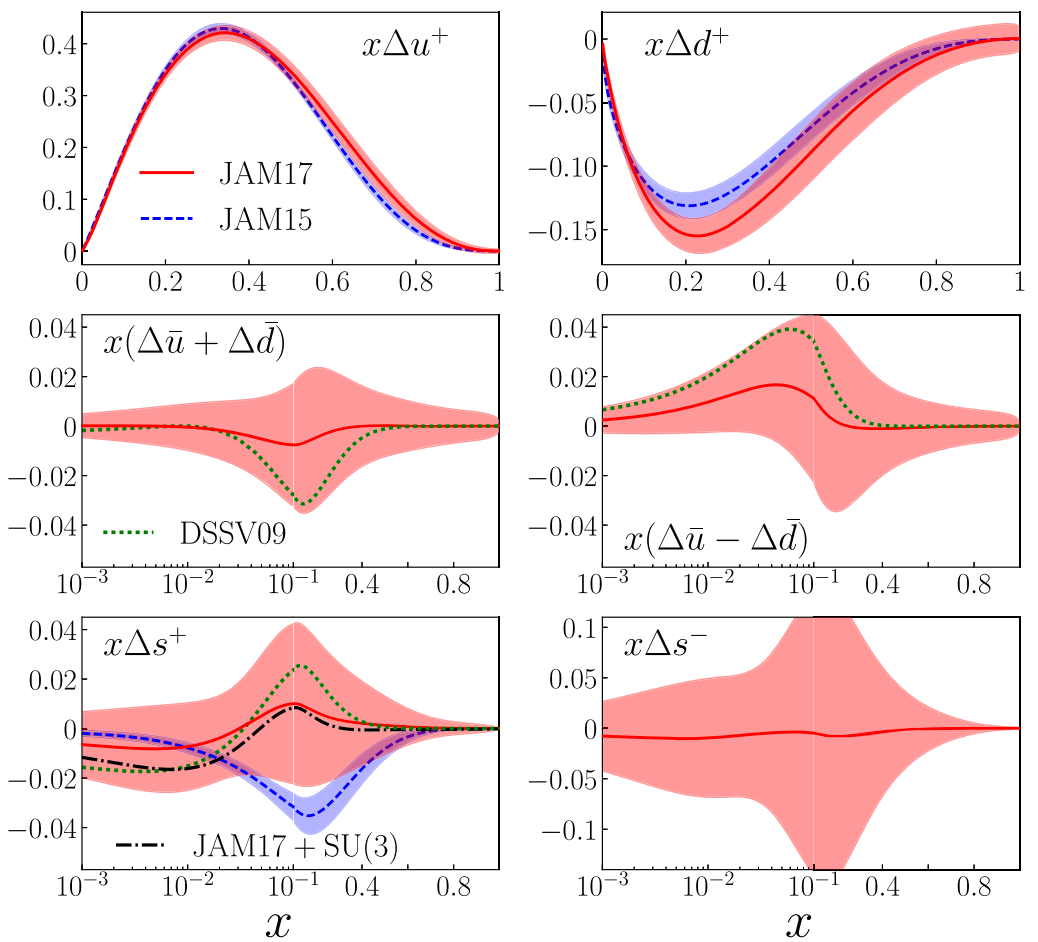

Figure 1: Spin-dependent PDFs at $Q=1 \mathrm{GeV}$ with $1 \sigma$ uncertainty bands from the fit by the JAM Collaboration [5].

jet productions measured by the STAR Collaboration $[6,7,8,9]$ and pion productions measured by the PHENIX Collaboration [10,11, 12, 13, 14, 15] have been incorporated into the global QCD analysis of spin-dependent PDFs. The first measurement of the longitudinal double spin asymmetry for midrapidity dijet production in polarized $p p$ collisions is reported by STAR Collaboration [16], shown in Figure 2. The measured asymmetry is consistent with the theoretical calculations using previous next-to leading order (NLO) global fit of polarized PDFs, and supports the finding of positive gluon polarization at the level of $\Delta G \sim 0.2$ over the region of $x>0.05[17,18]$. On the other hand, the first lattice QCD calculation of the gluon spin contribution to the nucleon spin is reported by the $\chi \mathrm{QCD}$ Collaboration [19]. The gluon spin in the Coulomb gauge in the $\overline{\mathrm{MS}}$ scheme is obtained from the one-loop perturbative matching. In the large momentum limit and at the physical pion mass, it is found that the gluon spin is $S_{G}=0.251(47)(16)$ at $\mu^{2}=10 \mathrm{GeV}^{2}$.

Although the proton spin-dependent structure function has been precisely measured in the DIS regime over a wide $x$-range, there are very few studies of the low- $Q^{2}$ region governed by soft processes and the transition region to high- $Q^{2}$. A measurement of the longitudinal double spin asymmetry and the spin structure function $g_{1}$ at low- $Q^{2}$ and small- $x$ region on a proton target using two beam energies $160 \mathrm{GeV}$ and $200 \mathrm{GeV}$ is reported by the COMPASS Collaboration [20], shown in Figure 3. The statistical precision is more than ten times better than previous measurements in this region. A positive asymmetry is observed, for the first time indicating the spin effects at such small $x$ values, while in the previously reported measurement on a deuteron target the asymmetry is compatible with zero [21]. 

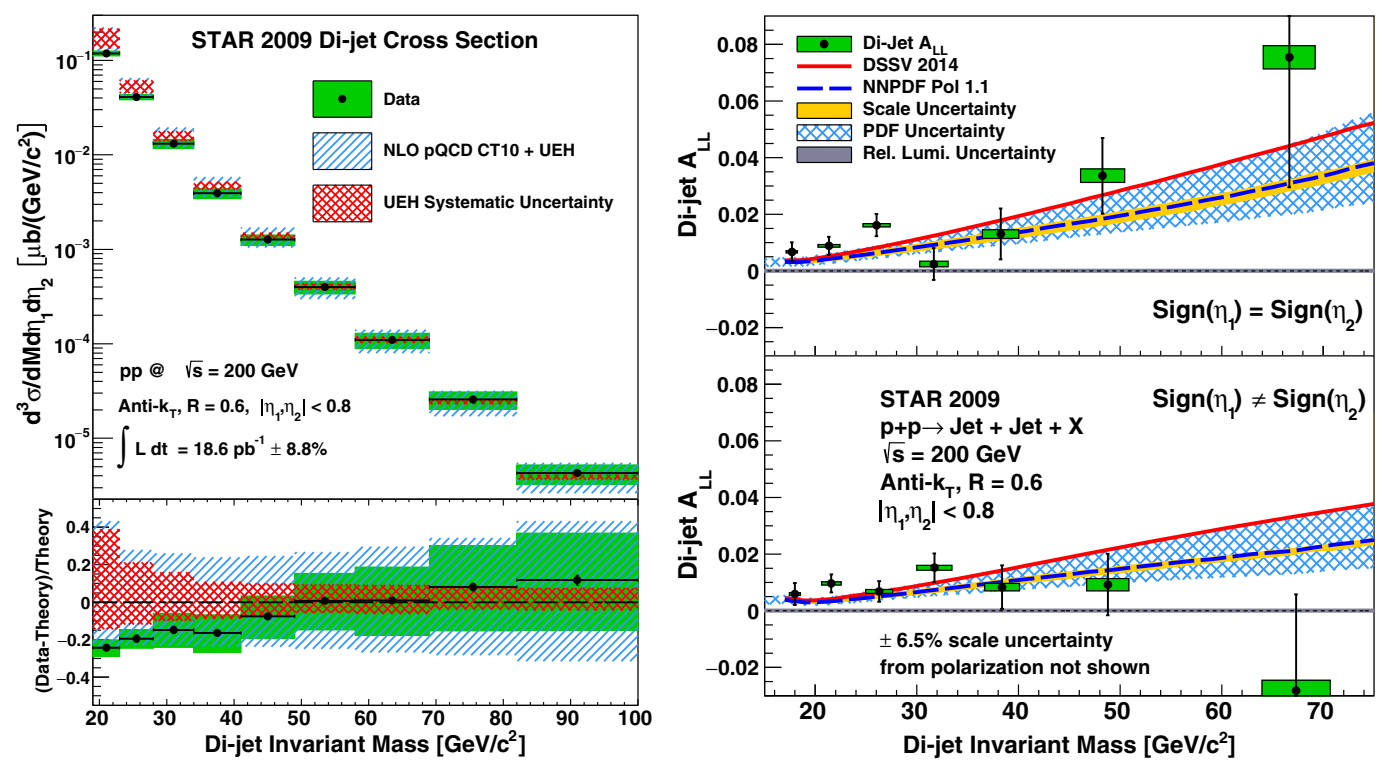

Figure 2: The cross section (left) and double spin asymmetry (right) of the midrapidity dijet production in $p p$ collisions measured by STAR Collaboration[16].
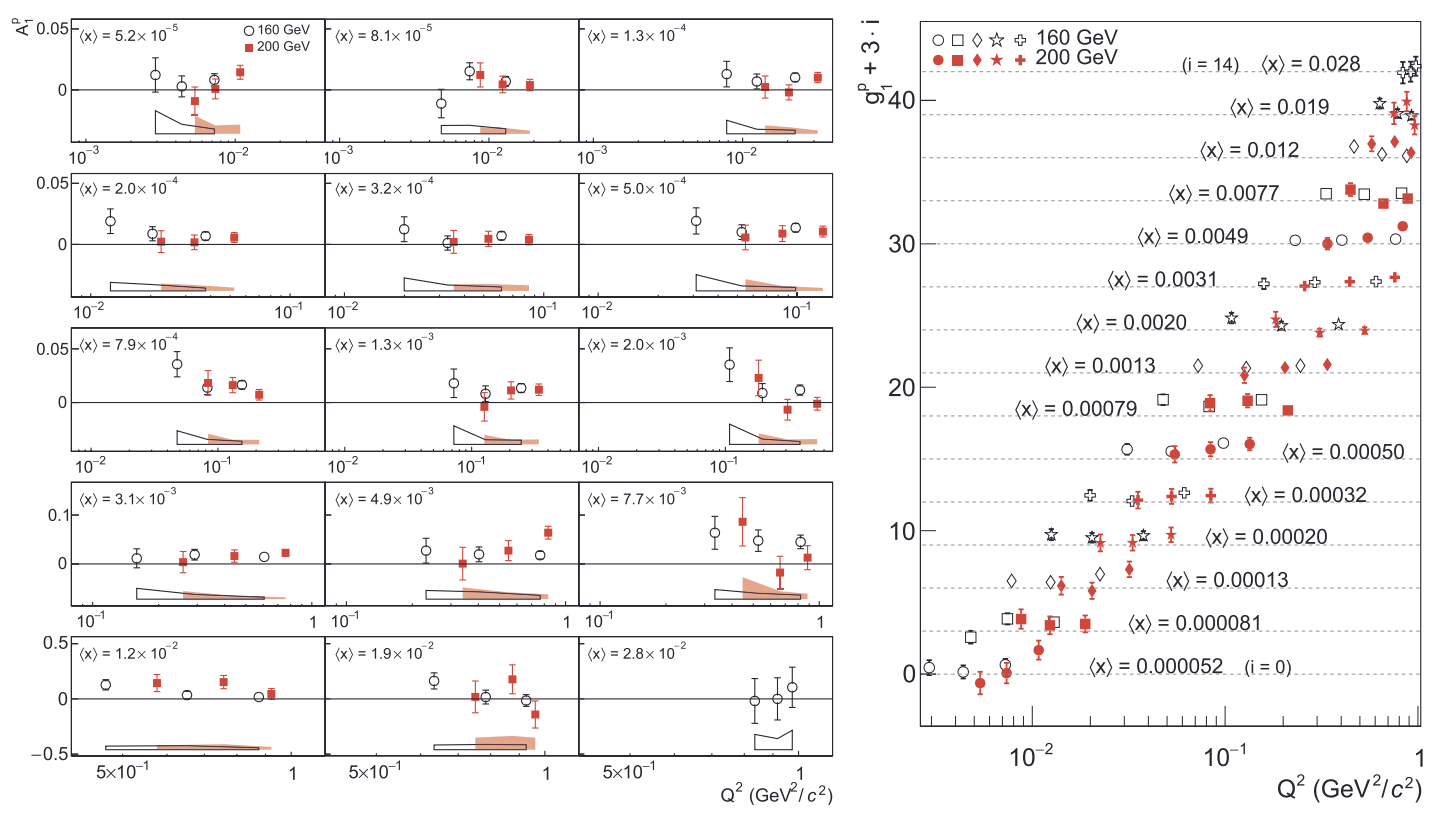

Figure 3: The longitudinal double spin asymmetry $A_{1}$ (left) and the spin dependent structure function $g_{1}$ (right) of the proton measured by COMPASS Collaboration[20]. 
The Gerasimov-Drell-Hearn (GDH) sum rule [22, 23] connects the spin-dependent total photoabsorption cross section of a particle to its anomalous magnetic moment $\kappa$. For a nucleon it is expressed as

$$
I_{N}^{\mathrm{GDH}}=\int_{v_{t h}}^{\infty} \frac{d v}{v}\left[\sigma_{\frac{3}{2}}(v)-\sigma_{\frac{1}{2}}(v)\right]=2 \pi^{2} \alpha \frac{\kappa^{2}}{M^{2}},
$$

where $\sigma_{\frac{3}{2}}$ and $\sigma_{\frac{1}{2}}$ are helicity dependent total photoabsorption cross sections with total helicity projection of $3 / 2$ and $1 / 2$ respectively, and for a nuclear target with spin- $S$, it is expressed as

$$
I^{\mathrm{GDH}}=\int_{v_{t h}}^{\infty} \frac{d v}{v}\left[\sigma_{P}(v)-\sigma_{A}(v)\right]=4 \pi^{2} \alpha \frac{\kappa^{2}}{M^{2}} S,
$$

where the subscripts $P$ and $A$ denote the beam helicity parallel and antiparallel to the target spin.

The GDH sum rule can be generalized to the virtual photon, known as the generalized GDH sum rule, which is related to the moment of the spin-dependent structure functions as

$$
\begin{aligned}
I_{T T}\left(Q^{2}\right) & =\frac{M^{2}}{4 \pi^{2} \alpha} \int_{v_{t h}}^{\infty} \frac{K\left(v, Q^{2}\right) \sigma_{T T}\left(v, Q^{2}\right)}{v^{2}} d v \\
& =\frac{2 M^{2}}{Q^{2}} \int_{0}^{x_{t h}}\left[g_{1}\left(x, Q^{2}\right)-\frac{4 M^{2}}{Q^{2}} x^{2} g_{2}\left(x, Q^{2}\right)\right] d x,
\end{aligned}
$$

where $K\left(v, Q^{2}\right)$ is the virtual photon flux.

The final analysis of the spin structure function of the proton using polarized electrons with energies of $1.6,2.5,4.2$, and $5.7 \mathrm{GeV}$ scattered from ${ }^{15} \mathrm{NH}_{3}$ targets (dynamically polarized along the beam direction) is reported by the CLAS Collaboration [24]. The double spin asymmetries are measured over a wide kinematic range $0.05 \mathrm{GeV}^{2}<Q^{2}<5 \mathrm{GeV}^{2}$ and $1.08 \mathrm{GeV}<W<3 \mathrm{GeV}$. The results of the moments of $g_{1}^{p}$ is shown in Figure 4. The preliminary result of the first moment of the neutron spin dependent structure function from the measurement in E97-110 at JLab using a high pressure polarized ${ }^{3} \mathrm{He}$ target [25] is also shown in Figure 4.

A precise measurement of the deuteron spin structure function and its moments at low- $Q^{2}$ is reported by the CLAS Collaboration [26]. The generalized GDH sum, shown in Figure 5, is precisely determined down to a minimum $Q^{2}$ of $0.02 \mathrm{GeV}^{2}$ for the first time, about 2.5 times lower than previously published data. Apart from the GDH sum, the forward spin polarizability, which is a higher moment of the spin structure functions,

$$
\gamma_{0}\left(Q^{2}\right)=\frac{16 \alpha M^{2}}{Q^{6}} \int_{0}^{x_{t h}} x^{2}\left[g_{1}\left(x, Q^{2}\right)-\frac{4 M^{2}}{Q^{2}} x^{2} g_{2}\left(x, Q^{2}\right)\right] d x,
$$

is also extracted as shown in Figure 5. The measurement at low- $Q^{2}$ provides a robust test of the chiral perturbation theory. While chiral calculations are reaching high precision, a satisfactory description of spin dependent observables still remains challenging.

\section{Progress in 3D structures}

In contrast to the spin terms which have been extensively studied, our knowledge of the orbital angular momentum terms in the nucleon spin decomposition is very little. As the orbital angular 

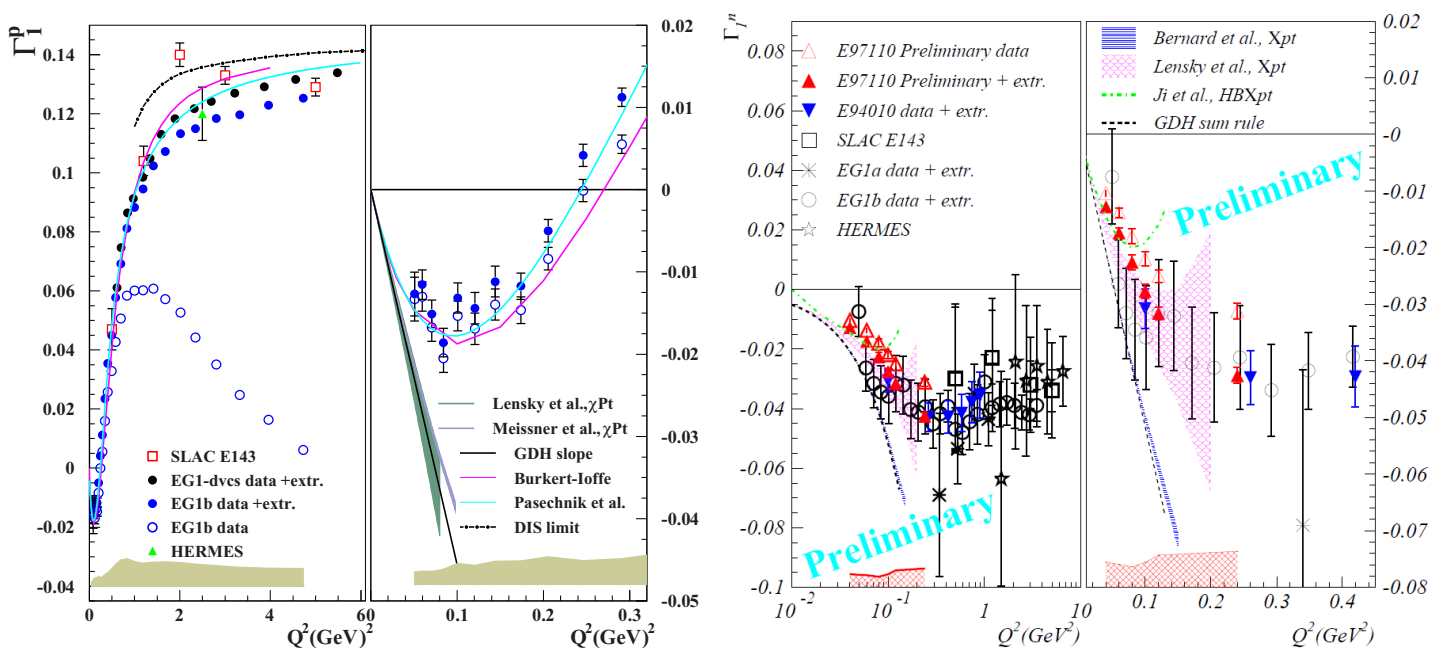

Figure 4: The first moment of the spin dependent structure function $g_{1}$ of the proton (left) [24] and the neutron (right) [25].
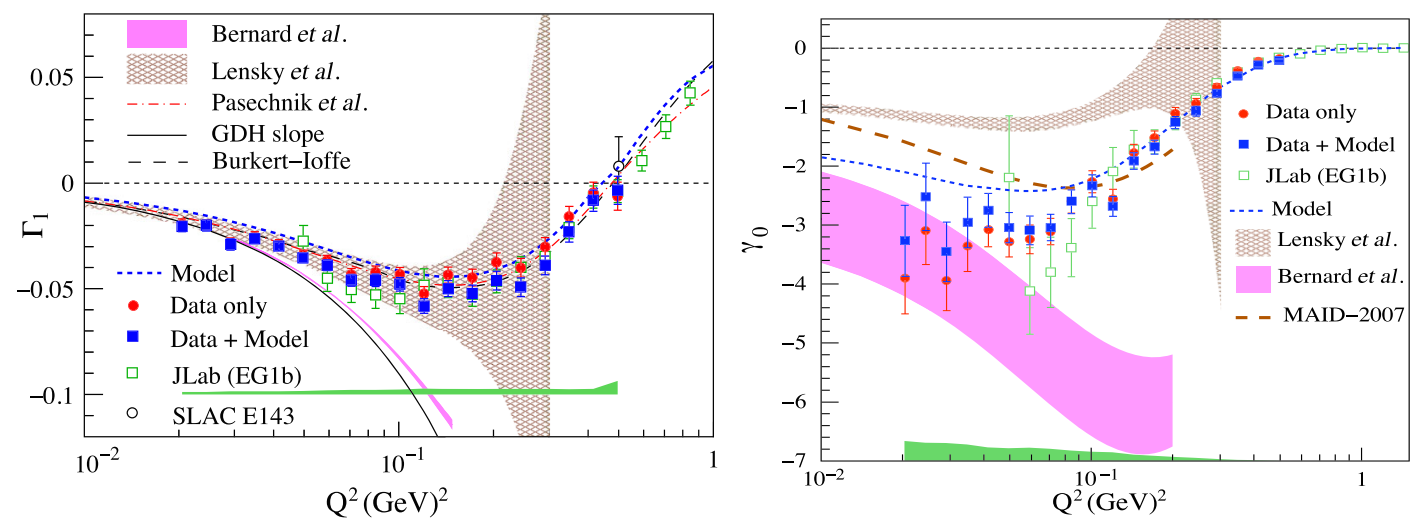

Figure 5: The GDH sum (left) and the forward spin polarizability (right) of the deuteron measured by CLAS Collaboration [26].

momentum contribution to the nucleon longitudinal spin is essentially the correlation between the transverse momentum and transverse position of quarks and gluons inside a polarized proton, one need to have $3 \mathrm{D}$ images of the nucleon through transverse momentum dependent parton distributions (TMDs) and generalized parton distributions (GPDs).

The SIDIS, Drell-Yan, and $e^{+} e^{-}$annihilation are primary processes to investigate TMDs. A simultaneous fit of SIDIS, Drell-Yan and $Z$ production data within the TMD formalism has been performed to extract unpolarized TMDs [27], which is the basis for the extraction of polarized TMDs. Although a precise determination of polarized TMDs are not possible due to the limited accuracy of the existing data, many explorations have been made [28, 29, 30].

The "golden channel" to measure GPDs is the deeply virtual Compton scattering (DVCS) process. It up to now provides the cleanest information about GPDs. In DVCS, GPDs contribute to the Compton form factors via weighted integrals, but only the cross-over line, where the longitudinal momentum fraction $x$, being equal to the skewness variable $\xi$, is directly measured. Another widely 
used process to access GPDs is the deeply virtual meson production (DVMP) process in which the GPD convolutes with the meson distribution amplitude. The double DVCS and the timelike Compton scattering (TCS) processes are complementary approaches to measure GPDs. Due to the lack of a multidimensional scan, the extraction of GPDs is usually based on some assumptions inspired by model calculations. Some explorations have been performed to fit GPDs with the existing world data $[31,32,33,34]$.

At the leading twist, eight quark TMDs are defined, and among them there are two naively time reversal odd (T-odd) TMDs: the Sivers function and the Boer-Mulders function, which arise from nontrivial Wilson lines that ensure the gauge invariance of QCD. One important prediction of the TMD factorization is the sign change of quark T-odd TMDs in SIDIS and Drell-Yan processes.

The first measurement of transverse spin dependent azimuthal asymmetries in the pion induced Drell-Yan process is reported by COMPASS Collaboration. Three single spin asymmetries giving access to different TMDs are extracted, shown in Figure 6. Comparing with theoretical calculations using the Sivers function extracted from SIDIS data, the asymmetry in $\pi N$ Drell-Yan data is consistent with the sign change prediction within the uncertainties, shown in Figure 6.
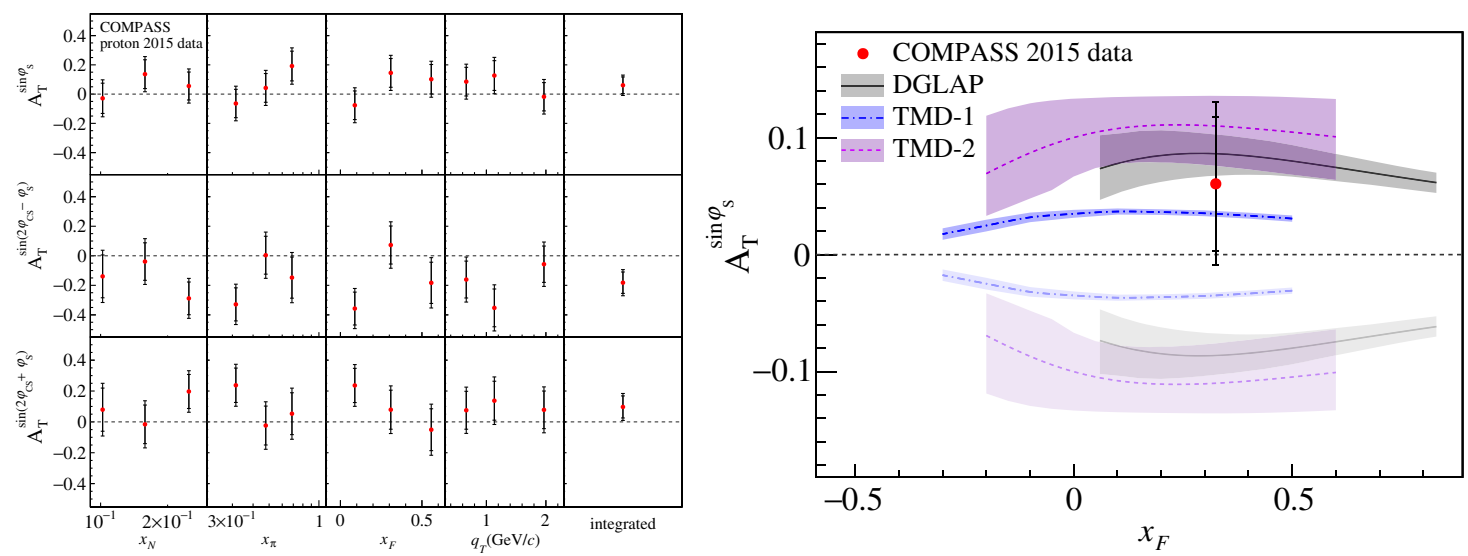

Figure 6: Transverse spin asymmetries (left) in $\pi N$ Drell-Yan process measured by the COMPASS Collaboration, and the comparison of the Sivers asymmetry with theoretical predictions (right) [35].

The first measurement of the Sivers asymmetry for gluons from SIDIS data are reported by the COMPASS Collaboration [36]. The analysis is based on a Monte Carlo simulation including three hard processes: photon-gluon fusion, QCD Compton scattering, and leading order virtual photon absorption as illustrated in Figure 7. A negative value is obtained for the gluon Sivers asymmetry with a significance of more than $2 \sigma$ deviations, shown in Figure 8. The result of a similar analysis of the gluon Collins-like asymmetry is consistent with zero.

The first measurement of transverse single spin asymmetries for inclusive jet and jet $+\pi^{ \pm}$ production at midrapidity from $p p$ collisions is reported by the STAR Collaboration. The Collins asymmetry, which is sensitive to quark transversity distribution coupled to the Collins fragmentation function, and the Collins-like asymmetry, which is sensitive to linearly polarized gluon distributions, are extracted, shown in Figure 9. At higher jet transverse momenta, nonzero Collins asymmetries in polarized $p p$ collisions are firstly observed with a statistical significance of more than $5 \sigma$. 


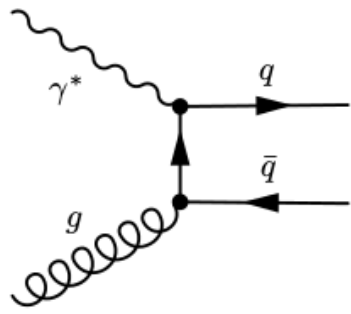

(a)

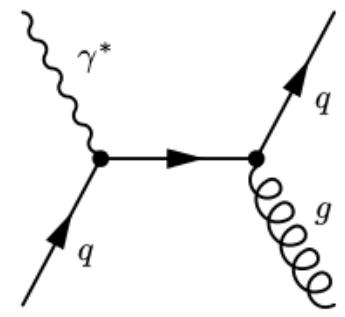

(b)

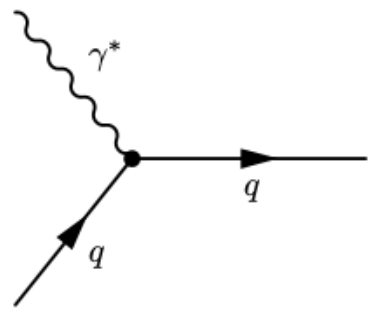

(c)

Figure 7: Hard processes considered in the analysis of the Sivers asymmetry for gluons by the COMPASS Collaboration [36]. They are (a) photon-gluon fusion (PGF), (b) QCD Compton scattering (QCDC), and (c) leading order process (LP).
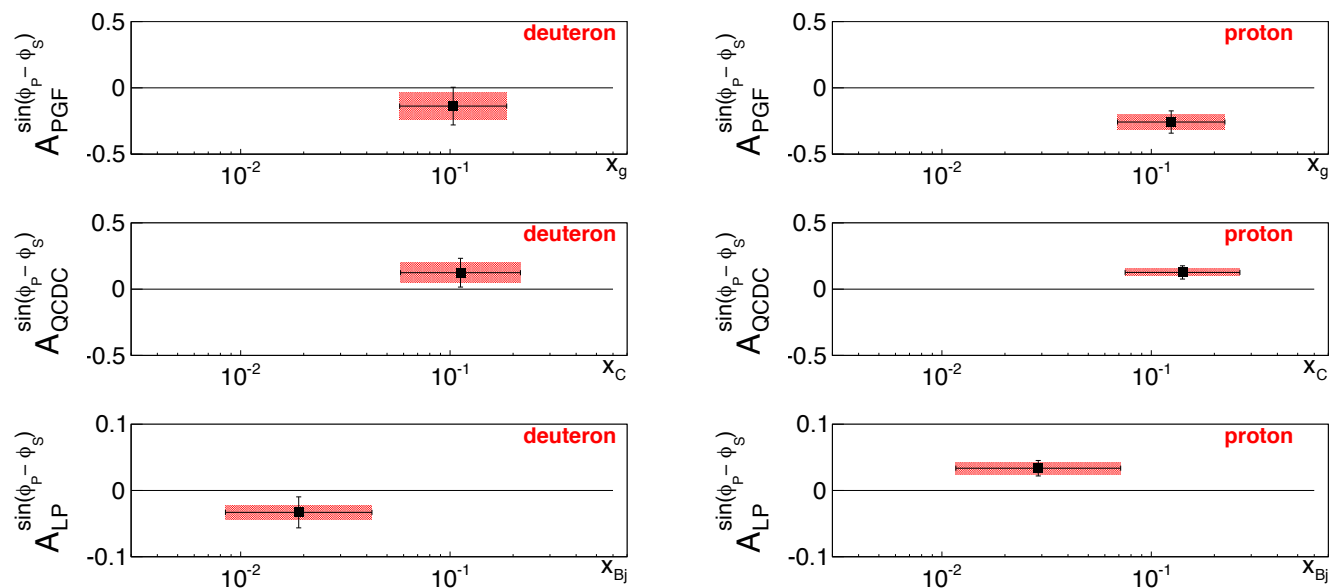

Figure 8: Sivers asymmetries of the deuteron (left) and the proton (right) extracted for PGF, QCDC, and LP subprocesses analyzed by the COMPASS Collaboration [36].
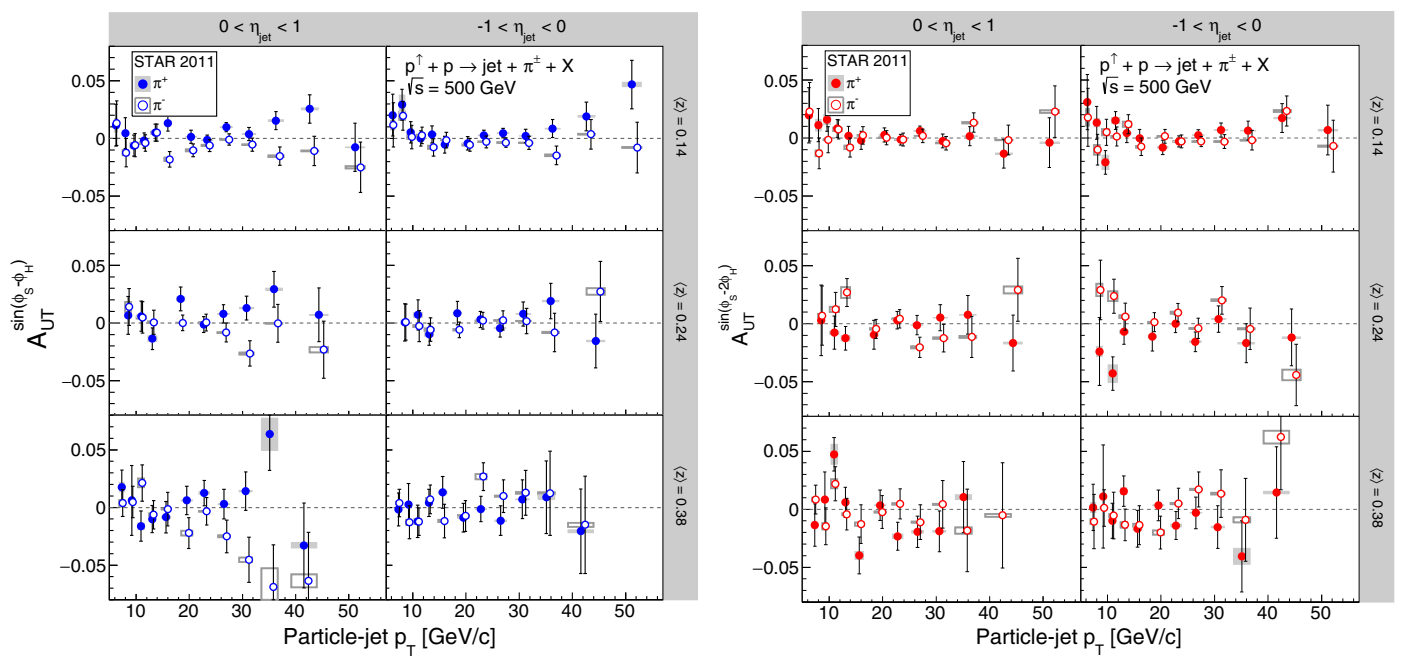

Figure 9: Collins (left) and Collins-like asymmetries for inclusive jet $+\pi^{ \pm}$in polarized $p p$ collisions measured by the STAR Collaboration [37]. 


\section{Future experiment}

After 30 years of measurements, the nucleon spin structure is still puzzling the physics community. Unveiling the nucleon spin structure and 3D images is one of the main goals at $12 \mathrm{GeV}$

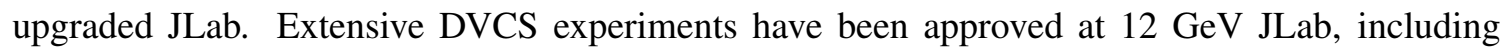
E12-06-114 with unpolarized proton target in Hall A, E12-06-119 with unpolarized and longitudinally polarized proton targets in Hall B, E12-11-003 with unpolarized neutron target in Hall B, E12-12-010 with transversely polarized proton target in Hall B, and E12-13-010 with unpolarized proton target in Hall C. The combination of experiments with the detector setups in different Halls and with different beam energies will provide a wide kinematic coverage. The SoLID spin program [38] in Hall A will combine large acceptance and high luminosities aiming at unprecedented precise measurement of nucleon 3D structures in the valence quark region.

\section{Summary}

The spin structure of the nucleon remains important and puzzling in nuclear and particle physics. The measurement of the three-dimensional imaging of the nucleon will help solve this remaining puzzle and uncover the rich dynamics of QCD. Major progresses have been made in spin and three-dimensional structure of the nucleon worldwide, and new experimental results on nucleon spin, TMDs and GPDs are expected in the near future, especially from those planned at 12 GeV JLab.

\section{References}

[1] J. Ashman et al. (European Muon Collaboration), Phys. Lett. B 206 (1988) 364.

[2] J. Ashman et al. (European Muon Collaboration), Nucl. Phys. B 328 (1989) 1.

[3] E. Leader and C. Lorcé, Phys. Rep. 541 (2014) 163.

[4] M. Wakamatsu, Int. J. Mod. Phys. A 29 (2014) 1430012.

[5] J. J. Ethier, N. Sato and W. Melnitchouk, Phys. Rev. Lett. 119 (2017) 132001.

[6] B. I. Abelev et al. (STAR Collaboration), Phys. Rev. Lett. 97 (2006) 252001.

[7] B. I. Abelev et al. (STAR Collaboration), Phys. Rev. Lett. 100 (2008) 232003.

[8] L. Adamczyk et al. (STAR Collaboration), Phys. Rev. D 86 (2012) 032006.

[9] L. Adamczyk et al. (STAR Collaboration), Phys. Rev. Lett. 115 (2015) 092002.

[10] S. S. Adler et al. (PHENIX Collaboration), Phys. Rev. D 73 (2006) 091102.

[11] A. Adare et al. (PHENIX Collaboration), Phys. Rev. D 76 (2007) 051106.

[12] A. Adare et al. (PHENIX Collaboration), Phys. Rev. Lett. 103 (2009) 012003.

[13] A. Adare et al. (PHENIX Collaboration), Phys. Rev. D 79 (2009) 012003.

[14] A. Adare et al. (PHENIX Collaboration), Phys. Rev. D 90 (2014) 012007.

[15] A. Adare et al. (PHENIX Collaboration), Phys. Rev. D 94 (2016) 112008. 
[16] L. Adamczyk et al. (STAR Collaboration), Phys. Rev. D 95 (2017) 071103.

[17] D. de Florian, R. Sassot, M. Stratmann and W. Vogelsang, Phys. Rev. Lett. 113 (2014) 012001.

[18] E. R. Nocera et al. (NNPDF Collaboration), Nucl. Phys. B 887 (2014) 276.

[19] Y. B. Yang, R. S. Sufian, A. Alexandru, T. Draper, M. J. Glatzmaier, K. F. Liu and Y. Zhao, Phys. Rev. Lett. 118 (2017) 102001.

[20] M. Aghasyan et al. (COMPASS Collaboration), Phys. Lett. B 781 (2018) 464.

[21] E. S. Ageev et al. (Compass Collaboration), Phys. Lett. B 647 (2007) 330.

[22] S. B. Gerasimov, Sov. J. Nucl. Phys. 2 (1966) 430 [Yad. Fiz. 2 (1965) 598].

[23] S. D. Drell and A. C. Hearn, Phys. Rev. Lett. 16 (1966) 908.

[24] R. Fersch et al. (CLAS Collaboration), Phys. Rev. C 96 (2017) 065208.

[25] V. Sulkosky et al., in preparation.

[26] K. P. Adhikari et al. (CLAS Collaboration), Phys. Rev. Lett. 120 (2018) 062501.

[27] A. Bacchetta, F. Delcarro, C. Pisano, M. Radici and A. Signori, J. High Energy Phys. 06 (2017) 081.

[28] M. Anselmino, M. Boglione, U. D’Alesio, A. Kotzinian, S. Melis, F. Murgia, A. Prokudin and C. Turk, Eur. Phys. J. A 39 (2009) 89.

[29] M. Anselmino, M. Boglione, U. D’Alesio, J. O. Gonzalez Hernandez, S. Melis, F. Murgia and A. Prokudin, Phys. Rev. D 92 (2015) 114023.

[30] C. Lefky and A. Prokudin, Phys. Rev. D 91 (2015) 034010.

[31] K. Kumeri?ki and D. Mueller, Nucl. Phys. B 841 (2010) 1.

[32] S. V. Goloskokov and P. Kroll, Eur. Phys. J. C 53 (2008) 367.

[33] G. R. Goldstein, J. O. Hernandez and S. Liuti, Phys. Rev. D 84 (2011) 034007.

[34] K. Kumericki and D. Müller, EPJ Web Conf. 112 (2016) 01012.

[35] M. Aghasyan et al. (COMPASS Collaboration), Phys. Rev. Lett. 119 (2017) 112002.

[36] C. Adolph et al. (COMPASS Collaboration), Phys. Lett. B 772 (2017) 854.

[37] L. Adamczyk et al. (STAR Collaboration), Phys. Rev. D 97 (2018) 032004.

[38] J. P. Chen et al. (SoLID Collaboration), arXiv:1409.7741 [nucl-ex]. 